\title{
Strong convergence of a new iterative algorithm for fixed points of asymptotically nonexpansive mappings
}

\section{Yuanheng Wang*, Jialei Feng}

Department of Mathematics, Zhejiang Normal University, Jinhua, China.

Communicated by Y. H. Yao

\begin{abstract}
In this paper, we investigate a new iterative implicit algorithm for fixed points of asymptotically nonexpansive mapping in Hilbert spaces. We also prove its strong convergence theorem under certain assumptions imposed on the parameters and extend some well-known results. As an application, we apply our main result to $\mu$-inverse strongly monotone mapping.
\end{abstract}

Keywords: Asymptotically nonexpansive, strong convergence, $\mu$-inverse strongly monotone mapping, Hilbert space.

2010 MSC: 47H09, 47H10, 54H25.

(C)2018 All rights reserved.

\section{Introduction}

In the theories of this paper, we suppose that $\mathrm{H}$ is a Hilbert space with the inner product $\langle\cdot, \cdot\rangle$ and norm $\|\cdot\|$. Let $C$ be a nonempty convex closed subset of $H$, and " $\rightarrow$ " and " $\rightarrow$ " denote the weak convergence and strong convergence, respectively. For a mapping $T: C \rightarrow C$, we use $F(T)$ and $N(T)$ to denote its fixed point set and zero point set, respectively.

Recall that the mapping $\mathrm{T}$ is said to be nonexpansive if

$$
\|\mathrm{T} x-\mathrm{T} y\| \leqslant\|x-y\|, \quad \forall x, y \in C .
$$

We call $\mathrm{T}$ is said to be attracting nonexpansive if it is nonexpansive and satisfies:

$$
\|T x-y\|<\|x-y\|, \quad \forall x \notin F(T), y \in F(T) .
$$

The mapping $T$ is said to be asymptotically nonexpansive if there exists a sequence $\left\{k_{n}\right\} \subset[1,+\infty)$ with $k_{n} \rightarrow 1$, such that

$$
\left\|T^{n} x-T^{n} y\right\| \leqslant k_{n}\|x-y\|, \quad \forall x, y \in C .
$$

The mapping $\mathrm{U}: \mathrm{C} \rightarrow \mathrm{H}$ is said to be monotone if

$$
\langle\mathrm{U} x-\mathrm{U} y, x-y\rangle \geqslant 0, \quad \forall x, y \in \mathrm{C} .
$$

\footnotetext{
*Corresponding author

Email addresses: yhwang@zjnu.cn (Yuanheng Wang), 1397638728@qq.com (Jialei Feng)

doi: $10.22436 /$ jnsa.011.04.09
}

Received: 2017-11-10 Revised: 2017-12-01 Accepted: 2018-01-26 
The mapping $\mathrm{U}: \mathrm{C} \rightarrow \mathrm{H}$ is said to be $\mu$-inverse strongly monotone if

$$
\langle\mathrm{U} x-\mathrm{U} y, x-\mathrm{y}\rangle \geqslant \mu\|\mathrm{U} x-\mathrm{U} y\|^{2}, \quad \forall x, y \in \mathrm{C},
$$

where $\mu>0$ is a positive real number.

Lately, in order to find a common element of the fixed points of nonexpansive mappings or asymptotically nonexpansive mappings, many authors established many viscosity iterative algorithms, and they solved many practical problems, such as the equilibrium problems, variational inequality problems, and split feasibility problems and so on. See $[1,2,4,9-14,21-24]$ and the references therein.

Recently, Moudafi [8] in Hilbert space introduced the viscosity iterative algorithm of nonexpansive. $\mathrm{Xu}$ [18] extended the Moudafi's results to more general uniformly smooth spaces. That is, he introduced the following explicit viscosity algorithm:

$$
x_{n+1}=\alpha_{n} f\left(x_{n}\right)+\left(1-\alpha_{n}\right) T x_{n},
$$

where $T$ is a nonexpansive mapping, $f$ is a strict contraction mapping, and $\left\{\alpha_{n}\right\}$ is a real number sequence in $(0,1)$. Then the sequence $\left\{x_{n}\right\}$ generated by (1.1) converges strongly to a fixed point of $T$.

In 2008, Lou et al. [7] showed the existence of the sequence $\left\{x_{n}\right\}$ defined by

$$
x_{n+1}=\alpha_{n} f\left(x_{n}\right)+\left(1-\alpha_{n}\right) T^{n} x_{n}
$$

converges strongly to the fixed point set of an asymptotically nonexpansive mapping $T$, which solves some variational inequality problems under suitable conditions. As an application, they proved that the iterative process defined by

$$
x_{0} \in C, x_{n+1}=\alpha_{n} f\left(x_{n}\right)+\beta_{n} x_{n}+\gamma_{n} T^{n} x_{n},
$$

converges strongly to the same fixed point of $\mathrm{T}$.

In 2015, Xu et al. [19] studied the following viscosity implicit midpoint rule:

$$
x_{n+1}=\alpha_{n} f\left(x_{n}\right)+\left(1-\alpha_{n}\right) T\left(\frac{x_{n}+x_{n+1}}{2}\right), n \geqslant 0 .
$$

They proved that the $\left\{x_{n}\right\}$ generated by (1.2) converges in norm to a fixed point of $T$.

Recently, Fan et al. [3] studied the following iterative algorithm:

$$
\left\{\begin{array}{l}
x_{n+1}=\left(1-\beta_{n}\right)\left(\lambda_{n} x_{n}\right)+\beta_{n} y_{n}, \\
y_{n}=\left(1-\alpha_{n}\right) x_{n}+\alpha_{n} T x_{n} .
\end{array}\right.
$$

They proved that the $\left\{x_{n}\right\}$ generated by (1.3) converges strongly to a fixed point $\hat{x}$ of nonexpansive mapping $T$, where $\hat{x}$ is the minimum-norm element of $F(T)$.

In this paper, motivated and inspired by the above authors' results, we give a new implicit iterative algorithm, to approximate fixed points of asymptotically nonexpansive mapping and prove its strong convergence theorem. As applications, we can use our main results to a family of $\mu$-inverse strongly monotone mappings for the common zeros.

\section{Preliminaries}

Definition 2.1. Let $\mathrm{C} \subset \mathrm{H}$ be a nonempty convex closed set of a Hilbert space $\mathrm{H}$, we define $\mathrm{P}_{\mathrm{C}}: \mathrm{C} \rightarrow \mathrm{H}$ be the nearest point projection if

$$
\left\|x-P_{C} x\right\| \leqslant\|x-y\|, \quad \forall y \in C .
$$


Lemma 2.2 ([6]). In a real Banach space $\mathrm{E}$, for $\mathrm{q}>1, \forall x, y \in \mathrm{E}$, the following inequality holds

$$
\|x+y\|^{\mathbf{q}} \leqslant\|x\|^{\mathbf{q}}+q\left\langle y, j_{\mathbf{q}}(x+y)\right\rangle, \forall j_{\mathbf{q}}(x+y) \in J_{\mathbf{q}}(x+y) .
$$

When $\mathrm{E}$ is a Hilbert space and $\mathrm{q}=2,(2.1)$ can be reduced to the following inequality

$$
\|x+y\|^{2} \leqslant\|x\|^{2}+2\langle y, x+y\rangle, \quad \forall x, y \in H .
$$

Lemma 2.3 ([15]). Let $\mathrm{C} \subset \mathrm{H}$ be a nonempty bounded convex closed set of a Hilbert space $\mathrm{H}$. Let $\mathrm{T}: \mathrm{C} \rightarrow \mathrm{C}$ be a asymptotically nonexpansive mapping with $\mathrm{F}(\mathrm{T}) \neq \emptyset$. Suppose $\mathrm{x}_{\mathrm{n}} \rightarrow \mathrm{x}$ and $(\mathrm{I}-\mathrm{T}) \mathrm{x}_{\mathrm{n}} \rightarrow \mathrm{y}$, then $(\mathrm{I}-\mathrm{T}) \mathrm{x}=\mathrm{y}$.

Lemma 2.4 ([16, 17]). Let $\left\{a_{n}\right\}_{n=0}^{\infty}$ be a nonnegative real number sequence satisfying the property:

$$
a_{n+1} \leqslant\left(1-\delta_{n}\right) a_{n}+\delta_{n} \gamma_{n}, \quad n \geqslant 0,
$$

where $\left\{\delta_{\mathfrak{n}}\right\}_{\mathfrak{n}=0}^{\infty} \subset(0,1)$ and $\left\{\gamma_{\mathfrak{n}}\right\}_{\mathfrak{n}=0}^{\infty}$ satisfy:

(i) $\lim _{n \rightarrow \infty} \delta_{n}=0$ and $\sum_{n=0}^{\infty} \delta_{n}=+\infty$;

(ii) either $\limsup _{n \rightarrow \infty} \gamma_{n} \leqslant 0$ or $\sum_{n=0}^{\infty}\left|\delta_{n} \gamma_{n}\right|<+\infty$.

Then $\lim _{n \rightarrow \infty} a_{n}=0$.

\section{Main results}

Theorem 3.1. Let $\mathrm{C} \subset \mathrm{H}$ be a nonempty bounded convex closed set of a Hilbert space $\mathrm{H}$ and $\theta \in \mathrm{C}$. Let $\mathrm{T}: \mathrm{C} \rightarrow \mathrm{C}$ be a asymptotically nonexpansive mapping with a sequence $\left\{k_{n}\right\}$ such that $\mathrm{F}(\mathrm{T}) \neq \emptyset$. Suppose $\left\{\alpha_{n}\right\},\left\{\beta_{n}\right\},\left\{\lambda_{n}\right\},\left\{\xi_{n}\right\}$ are real number sequences in $(0,1)$. Let $\left\{x_{n}\right\}$ be generated by

$$
\left\{\begin{array}{l}
x_{1} \in C \\
y_{n}=\left(1-\alpha_{n}\right) x_{n}+\alpha_{n} T^{n}\left(\xi_{n} x_{n}+\left(1-\xi_{n}\right) x_{n+1}\right), \\
x_{n+1}=\left(1-\beta_{n}\right)\left(\lambda_{n} x_{n}\right)+\beta_{n} y_{n} .
\end{array}\right.
$$

Suppose the following conditions are satisfied:

(i) $\lim _{n \rightarrow \infty} \beta_{n}=1, \lim _{n \rightarrow \infty} \lambda_{n}=1$;

(ii) $\sum_{n=1}^{\infty} \alpha_{n}<+\infty, \sum_{n=1}^{\infty}\left|\lambda_{n+1}-\lambda_{n}\right|<+\infty, \sum_{n=1}^{\infty}\left|\beta_{n+1}-\beta_{n}\right|<+\infty$;

(iii) $\sum_{n=1}^{\infty}\left(1-\lambda_{n}\right)\left(1-\beta_{n}\right)=+\infty,\left|\lambda_{n+1}-\beta_{n} \lambda_{n}\right|+\beta_{n+1} \leqslant 1$;

(iv) $\sum_{n=1}^{\infty} \sup _{x \in C}\left\|T^{n+1} x-T^{n} x\right\|<+\infty$.

Then the sequence $\left\{x_{n}\right\}$ converges strongly to a fixed point $x^{*} \in F(T)$.

Proof. We divide the proof into four steps.

Step 1 . We show the sequence $\left\{x_{n}\right\}$ is bounded.

In fact, for $p \in F(T)$, we can easily know that

$$
\begin{aligned}
\left\|y_{n}-p\right\| & =\left\|\left(1-\alpha_{n}\right) x_{n}+\alpha_{n} T^{n}\left(\xi_{n} x_{n}+\left(1-\xi_{n}\right) x_{n+1}\right)-p\right\| \\
& =\left\|\left(1-\alpha_{n}\right)\left(x_{n}-p\right)+\alpha_{n}\left(T^{n}\left(\xi_{n} x_{n}+\left(1-\xi_{n}\right) x_{n+1}\right)-p\right)\right\| \\
& \left.\leqslant\left(1-\alpha_{n}\right)\left\|x_{n}-p\right\|+\alpha_{n} \| T^{n}\left(\xi_{n} x_{n}+\left(1-\xi_{n}\right) x_{n+1}\right)-p\right) \| \\
& \left.\leqslant\left(1-\alpha_{n}\right)\left\|x_{n}-p\right\|+\alpha_{n} k_{n} \| \xi_{n} x_{n}+\left(1-\xi_{n}\right) x_{n+1}-p\right) \| \\
& \leqslant\left(1-\alpha_{n}\right)\left\|x_{n}-p\right\|+\alpha_{n} k_{n} \xi_{n}\left\|x_{n}-p\right\|+\alpha_{n} k_{n}\left(1-\xi_{n}\right)\left\|x_{n+1}-p\right\| \\
& =\left(1-\alpha_{n}+\alpha_{n} k_{n} \xi_{n}\right)\left\|x_{n}-p\right\|+\alpha_{n} k_{n}\left(1-\xi_{n}\right)\left\|x_{n+1}-p\right\| .
\end{aligned}
$$


And then from (3.2),

$$
\begin{aligned}
\left\|x_{n+1}-p\right\|= & \left\|\left(1-\beta_{n}\right)\left(\lambda_{n} x_{n}\right)+\beta_{n} y_{n}-p\right\| \\
= & \left\|\left(1-\beta_{n}\right)\left(\lambda_{n} x_{n}-\lambda_{n} p+\lambda_{n} p-p\right)+\beta_{n}\left(y_{n}-p\right)\right\| \\
\leqslant & \left(1-\beta_{n}\right) \lambda_{n}\left\|x_{n}-p\right\|+\left(1-\beta_{n}\right)\left(1-\lambda_{n}\right)\|p\|+\beta_{n}\left\|y_{n}-p\right\| \\
\leqslant & \left(1-\beta_{n}\right) \lambda_{n}\left\|x_{n}-p\right\|+\left(1-\beta_{n}\right)\left(1-\lambda_{n}\right)\|p\| \\
& +\left(\beta_{n}-\alpha_{n} \beta_{n}+\alpha_{n} \beta_{n} k_{n} \xi_{n}\right)\left\|x_{n}-p\right\|+\alpha_{n} \beta_{n} k_{n}\left(1-\xi_{n}\right)\left\|x_{n+1}-p\right\| \\
= & \left(\lambda_{n}-\beta_{n} \lambda_{n}+\beta_{n}-\alpha_{n} \beta_{n}+\alpha_{n} \beta_{n} k_{n} \xi_{n}\right)\left\|x_{n}-p\right\| \\
& +\left(1-\beta_{n}\right)\left(1-\lambda_{n}\right)\|p\|+\alpha_{n} \beta_{n} k_{n}\left(1-\xi_{n}\right)\left\|x_{n+1}-p\right\|,
\end{aligned}
$$

which implies

$$
\begin{aligned}
\left\|x_{n+1}-p\right\| \leqslant & \frac{\lambda_{n}-\beta_{n} \lambda_{n}+\beta_{n}-\alpha_{n} \beta_{n}+\alpha_{n} \beta_{n} k_{n} \xi_{n}}{1-\alpha_{n} \beta_{n} k_{n}\left(1-\xi_{n}\right)}\left\|x_{n}-p\right\|+\frac{\left(1-\beta_{n}\right)\left(1-\lambda_{n}\right)}{1-\alpha_{n} \beta_{n} k_{n}\left(1-\xi_{n}\right)}\|p\| \\
= & \left(1-\frac{\left(1-\beta_{n}\right)\left(1-\lambda_{n}\right)-\alpha_{n} \beta_{n}\left(k_{n}-1\right)}{1-\alpha_{n} \beta_{n} k_{n}\left(1-\xi_{n}\right)}\right)\left\|x_{n}-p\right\| \\
& +\frac{\left(1-\beta_{n}\right)\left(1-\lambda_{n}\right)-\alpha_{n} \beta_{n}\left(k_{n}-1\right)+\alpha_{n} \beta_{n}\left(k_{n}-1\right)}{1-\alpha_{n} \beta_{n} k_{n}\left(1-\xi_{n}\right)}\|p\| \\
= & \left(1-\frac{\left(1-\beta_{n}\right)\left(1-\lambda_{n}\right)-\alpha_{n} \beta_{n}\left(k_{n}-1\right)}{1-\alpha_{n} \beta_{n} k_{n}\left(1-\xi_{n}\right)}\right)\left\|x_{n}-p\right\| \\
& +\frac{\left(1-\beta_{n}\right)\left(1-\lambda_{n}\right)-\alpha_{n} \beta_{n}\left(k_{n}-1\right)}{1-\alpha_{n} \beta_{n} k_{n}\left(1-\xi_{n}\right)}\|p\|+\frac{\alpha_{n} \beta_{n}\left(k_{n}-1\right)}{1-\alpha_{n} \beta_{n} k_{n}\left(1-\xi_{n}\right)}\|p\| .
\end{aligned}
$$

Let $M_{1}=\sup _{n \in \mathbb{N}}\left\{k_{n}-1\right\}$, then by conditions (i) and (ii), there exists $N \in \mathbb{N}$ such that for all $n \geqslant N$,

$$
\frac{\left(1-\beta_{n}\right)\left(1-\lambda_{n}\right)-\alpha_{n} \beta_{n}\left(k_{n}-1\right)}{1-\alpha_{n} \beta_{n} k_{n}\left(1-\xi_{n}\right)}<1
$$

and

$$
1-\alpha_{n} \beta_{n} k_{n}\left(1-\xi_{n}\right) \geqslant \frac{1}{2}
$$

So we have

$$
\begin{aligned}
\left\|x_{n+1}-p\right\| \leqslant & \left(1-\frac{\left(1-\beta_{n}\right)\left(1-\lambda_{n}\right)-\alpha_{n} \beta_{n}\left(k_{n}-1\right)}{1-\alpha_{n} \beta_{n} k_{n}\left(1-\xi_{n}\right)}\right)\left\|x_{n}-p\right\| \\
& +\frac{\left(1-\beta_{n}\right)\left(1-\lambda_{n}\right)-\alpha_{n} \beta_{n}\left(k_{n}-1\right)}{1-\alpha_{n} \beta_{n} k_{n}\left(1-\xi_{n}\right)}\|p\|+2 \alpha_{n} M_{1}\|p\| \\
\leqslant & \max \left\{\left\|x_{n}-p\right\|,\|p\|\right\}+2 \alpha_{n} M_{1}\|p\| \\
& \vdots \\
\leqslant & \max \left\{\left\|x_{1}-p\right\|,\|p\|\right\}+2 M_{1}\|p\| \sum_{n=1}^{\infty} \alpha_{n}<+\infty .
\end{aligned}
$$

Therefore, by condition (ii), we obtain that $\left\{x_{n}\right\}$ is bounded. So are $\left\{y_{n}\right\}$ and $\left\{T^{n}\left(\xi_{n} x_{n}+\left(1-\xi_{n}\right) x_{n+1}\right)\right\}$.

Step 2. We prove that $\left\|x_{n+1}-x_{n}\right\| \rightarrow 0$, as $n \rightarrow \infty$. 


\section{Indeed, we observe that}

$$
\begin{aligned}
y_{n+1}-y_{n}= & \left(1-\alpha_{n+1}\right) x_{n+1}+\alpha_{n+1} T^{n+1}\left(\xi_{n+1} x_{n+1}+\left(1-\xi_{n+1}\right) x_{n+2}\right)-\left(1-\alpha_{n}\right) x_{n} \\
& -\alpha_{n} T^{n}\left(\xi_{n} x_{n}+\left(1-\xi_{n}\right) x_{n+1}\right) \\
= & \left(1-\alpha_{n+1}\right) x_{n+1}-\left(1-\alpha_{n}\right) x_{n+1}+\left(1-\alpha_{n}\right) x_{n+1}-\left(1-\alpha_{n}\right) x_{n} \\
& +\alpha_{n+1} T^{n+1}\left(\xi_{n+1} x_{n+1}+\left(1-\xi_{n+1}\right) x_{n+2}\right)-\alpha_{n} T^{n+1}\left(\xi_{n+1} x_{n+1}+\left(1-\xi_{n+1}\right) x_{n+2}\right) \\
& +\alpha_{n} T^{n+1}\left(\xi_{n+1} x_{n+1}+\left(1-\xi_{n+1}\right) x_{n+2}\right)-\alpha_{n} T^{n}\left(\xi_{n} x_{n}+\left(1-\xi_{n}\right) x_{n+1}\right) \\
= & \left(1-\alpha_{n}\right)\left(x_{n+1}-x_{n}\right)-\left(\alpha_{n+1}-\alpha_{n}\right) x_{n+1} \\
& +\alpha_{n}\left(T^{n+1}\left(\xi_{n+1} x_{n+1}+\left(1-\xi_{n+1}\right) x_{n+2}\right)-T^{n}\left(\xi_{n} x_{n}+\left(1-\xi_{n}\right) x_{n+1}\right)\right) \\
& +\left(\alpha_{n+1}-\alpha_{n}\right) T^{n+1}\left(\xi_{n+1} x_{n+1}+\left(1-\xi_{n+1}\right) x_{n+2}\right) \\
= & \left(1-\alpha_{n}\right)\left(x_{n+1}-x_{n}\right)-\left(\alpha_{n+1}-\alpha_{n}\right) x_{n+1} \\
& +\alpha_{n}\left(T^{n}\left(\xi_{n+1} x_{n+1}+\left(1-\xi_{n+1}\right) x_{n+2}\right)-T^{n}\left(\xi_{n} x_{n}+\left(1-\xi_{n}\right) x_{n+1}\right)\right) \\
& +\left(\alpha_{n+1}-\alpha_{n}\right) T^{n+1}\left(\xi_{n+1} x_{n+1}+\left(1-\xi_{n+1}\right) x_{n+2}\right) \\
& +\alpha_{n}\left(T^{n+1}\left(\xi_{n+1} x_{n+1}+\left(1-\xi_{n+1}\right) x_{n+2}\right)-T^{n}\left(\xi_{n+1} x_{n+1}+\left(1-\xi_{n+1}\right) x_{n+2}\right)\right) .
\end{aligned}
$$

Then from (3.3)

$$
\begin{aligned}
\left\|y_{n+1}-y_{n}\right\| \leqslant & \left(1-\alpha_{n}\right)\left\|x_{n+1}-x_{n}\right\|+\left|\alpha_{n+1}-\alpha_{n}\right|\left\|x_{n+1}\right\| \\
& +\left|\alpha_{n+1}-\alpha_{n}\right|\left\|T^{n+1}\left(\xi_{n+1} x_{n+1}+\left(1-\xi_{n+1}\right) x_{n+2}\right)\right\| \\
& +\alpha_{n}\left\|T^{n}\left(\xi_{n+1} x_{n+1}+\left(1-\xi_{n+1}\right) x_{n+2}\right)-T^{n}\left(\xi_{n} x_{n}+\left(1-\xi_{n}\right) x_{n+1}\right)\right\| \\
& +\alpha_{n}\left\|T^{n+1}\left(\xi_{n+1} x_{n+1}+\left(1-\xi_{n+1}\right) x_{n+2}\right)-T^{n}\left(\xi_{n+1} x_{n+1}+\left(1-\xi_{n+1}\right) x_{n+2}\right)\right\| \\
\leqslant & \left(1-\alpha_{n}\right)\left\|x_{n+1}-x_{n}\right\|+\left|\alpha_{n+1}-\alpha_{n}\right|\left\|x_{n+1}\right\| \\
& +\left|\alpha_{n+1}-\alpha_{n}\right|\left\|T^{n+1}\left(\xi_{n+1} x_{n+1}+\left(1-\xi_{n+1}\right) x_{n+2}\right)\right\| \\
& +\alpha_{n} k_{n}\left\|\left(\xi_{n+1} x_{n+1}+\left(1-\xi_{n+1}\right) x_{n+2}\right)-\left(\xi_{n} x_{n}+\left(1-\xi_{n}\right) x_{n+1}\right)\right\| \\
& +\alpha_{n}\left\|T^{n+1}\left(\xi_{n+1} x_{n+1}+\left(1-\xi_{n+1}\right) x_{n+2}\right)-T^{n}\left(\xi_{n+1} x_{n+1}+\left(1-\xi_{n+1}\right) x_{n+2}\right)\right\| \\
\leqslant & \left(1-\alpha_{n}\right)\left\|x_{n+1}-x_{n}\right\|+\left|\alpha_{n+1}-\alpha_{n}\right|\left\|x_{n+1}\right\| \\
& +\left|\alpha_{n+1}-\alpha_{n}\right|\left\|T^{n+1}\left(\xi_{n+1} x_{n+1}+\left(1-\xi_{n+1}\right) x_{n+2}\right)\right\| \\
& +\alpha_{n}\left\|T^{n+1}\left(\xi_{n+1} x_{n+1}+\left(1-\xi_{n+1}\right) x_{n+2}\right)-T^{n}\left(\xi_{n+1} x_{n+1}+\left(1-\xi_{n+1}\right) x_{n+2}\right)\right\| \\
& +\alpha_{n} k_{n}\left(1-\xi_{n+1}\right)\left\|x_{n+2}-x_{n+1}\right\|+\alpha_{n} k_{n} \xi_{n}\left\|x_{n+1}-x_{n}\right\| \\
= & \left(1-\alpha_{n}+\alpha_{n} k_{n} \xi_{n}\right)\left\|x_{n+1}-x_{n}\right\|+\left|\alpha_{n+1}-\alpha_{n}\right|\left\|x_{n+1}\right\| \\
& +\left|\alpha_{n+1}-\alpha_{n}\right|\left\|T^{n+1}\left(\xi_{n+1} x_{n+1}+\left(1-\xi_{n+1}\right) x_{n+2}\right)\right\| \\
& +\alpha_{n} k_{n}\left(1-\xi_{n+1}\right)\left\|x_{n+2}-x_{n+1}\right\| \\
& +\alpha_{n}\left\|T^{n+1}\left(\xi_{n+1} x_{n+1}+\left(1-\xi_{n+1}\right) x_{n+2}\right)-T^{n}\left(\xi_{n+1} x_{n+1}+\left(1-\xi_{n+1}\right) x_{n+2}\right)\right\| .
\end{aligned}
$$

And also

$$
\begin{aligned}
x_{n+2}-x_{n+1}= & \left(1-\beta_{n+1}\right)\left(\lambda_{n+1} x_{n+1}\right)+\beta_{n+1} y_{n+1}-\left(1-\beta_{n}\right)\left(\lambda_{n} x_{n}\right)-\beta_{n} y_{n} \\
= & \lambda_{n+1} x_{n+1}-\beta_{n+1} \lambda_{n+1} x_{n+1}+\beta_{n+1} y_{n+1}-\lambda_{n} x_{n}+\beta_{n} \lambda_{n} x_{n}-\beta_{n} y_{n} \\
= & \lambda_{n+1} x_{n+1}-\lambda_{n+1} x_{n}+\lambda_{n+1} x_{n}-\lambda_{n} x_{n}-\beta_{n+1} \lambda_{n+1} x_{n+1}+\beta_{n} \lambda_{n} x_{n+1} \\
& -\beta_{n} \lambda_{n} x_{n+1}+\beta_{n} \lambda_{n} x_{n}+\beta_{n+1} y_{n+1}-\beta_{n+1} y_{n}+\beta_{n+1} y_{n}-\beta_{n} y_{n} \\
= & \lambda_{n+1}\left(x_{n+1}-x_{n}\right)+\left(\lambda_{n+1}-\lambda_{n}\right) x_{n}-\left(\beta_{n+1} \lambda_{n+1}-\beta_{n} \lambda_{n}\right) x_{n+1} \\
& -\beta_{n} \lambda_{n}\left(x_{n+1}-x_{n}\right)+\beta_{n+1}\left(y_{n+1}-y_{n}\right)+\left(\beta_{n+1}-\beta_{n}\right) y_{n} \\
= & \left(\lambda_{n+1}-\beta_{n} \lambda_{n}\right)\left(x_{n+1}-x_{n}\right)+\left(\lambda_{n+1}-\lambda_{n}\right) x_{n} \\
& -\left(\beta_{n+1} \lambda_{n+1}-\beta_{n} \lambda_{n}\right) x_{n+1}+\beta_{n+1}\left(y_{n+1}-y_{n}\right)+\left(\beta_{n+1}-\beta_{n}\right) y_{n} .
\end{aligned}
$$


Hence, it follows from (3.4) and (3.5),

$$
\begin{aligned}
\left\|x_{n+2}-x_{n+1}\right\| \leqslant & \left|\lambda_{n+1}-\beta_{n} \lambda_{n}\right|\left\|x_{n+1}-x_{n}\right\|+\left|\lambda_{n+1}-\lambda_{n}\right|\left\|x_{n}\right\| \\
& +\left|\beta_{n+1} \lambda_{n+1}-\beta_{n} \lambda_{n}\right|\left\|x_{n+1}\right\|+\beta_{n+1}\left\|y_{n+1}-y_{n}\right\|+\left|\beta_{n+1}-\beta_{n}\right|\left\|y_{n}\right\| \\
\leqslant & \left|\lambda_{n+1}-\beta_{n} \lambda_{n}\right|\left\|x_{n+1}-x_{n}\right\|+\left|\lambda_{n+1}-\lambda_{n}\right|\left\|x_{n}\right\| \\
& +\left|\beta_{n+1}-\beta_{n}\right|\left\|y_{n}\right\|+\left(\beta_{n+1}-\alpha_{n} \beta_{n+1}+\alpha_{n} \beta_{n+1} k_{n} \xi_{n}\right)\left\|x_{n+1}-x_{n}\right\| \\
& +\beta_{n+1}\left|\alpha_{n+1}-\alpha_{n}\right|\left\|x_{n+1}\right\|+\beta_{n+1}\left|\alpha_{n+1}-\alpha_{n}\right|\left\|T^{n+1}\left(\xi_{n+1} x_{n+1}+\left(1-\xi_{n+1}\right) x_{n+2}\right)\right\| \\
& +\alpha_{n} \beta_{n+1}\left\|T^{n+1}\left(\xi_{n+1} x_{n+1}+\left(1-\xi_{n+1}\right) x_{n+2}\right)-T^{n}\left(\xi_{n+1} x_{n+1}+\left(1-\xi_{n+1}\right) x_{n+2}\right)\right\| \\
& +\alpha_{n} \beta_{n+1} k_{n}\left(1-\xi_{n+1}\right)\left\|x_{n+2}-x_{n+1}\right\|+\left|\beta_{n+1} \lambda_{n+1}-\beta_{n} \lambda_{n}\right|\left\|x_{n+1}\right\| \\
= & \left(\left|\lambda_{n+1}-\beta_{n} \lambda_{n}\right|+\beta_{n+1}-\alpha_{n} \beta_{n+1}+\alpha_{n} \beta_{n+1} k_{n} \xi_{n}\right)\left\|x_{n+1}-x_{n}\right\| \\
& +\left|\lambda_{n+1}-\lambda_{n}\right|\left\|x_{n}\right\|+\left(\left|\beta_{n+1} \lambda_{n+1}-\beta_{n} \lambda_{n}\right|+\beta_{n+1}\left|\alpha_{n+1}-\alpha_{n}\right|\right)\left\|x_{n+1}\right\| \\
& +\left|\beta_{n+1}-\beta_{n}\right|\left\|y_{n}\right\|+\beta_{n+1}\left|\alpha_{n+1}-\alpha_{n}\right|\left\|T^{n+1}\left(\xi_{n+1} x_{n+1}+\left(1-\xi_{n+1}\right) x_{n+2}\right)\right\| \\
& +\alpha_{n} \beta_{n+1} \| T^{n+1}\left(\xi_{n+1} x_{n+1}+\left(1-\xi_{n+1}\right) x_{n+2}-T^{n}\left(\xi_{n+1} x_{n+1}+\left(1-\xi_{n+1}\right) x_{n+2}\right) \|\right. \\
& +\alpha_{n} \beta_{n+1} k_{n}\left(1-\xi_{n+1}\right)\left\|x_{n+2}-x_{n+1}\right\|,
\end{aligned}
$$

which implies

$$
\begin{aligned}
\left\|x_{n+2}-x_{n+1}\right\| \leqslant & \frac{\left|\lambda_{n+1}-\beta_{n} \lambda_{n}\right|+\beta_{n+1}-\alpha_{n} \beta_{n+1}+\alpha_{n} \beta_{n+1} k_{n} \xi_{n}}{1-\alpha_{n} \beta_{n+1} k_{n}\left(1-\xi_{n+1}\right)}\left\|x_{n+1}-x_{n}\right\| \\
& +\frac{\left|\lambda_{n+1}-\lambda_{n}\right|}{1-\alpha_{n} \beta_{n+1} k_{n}\left(1-\xi_{n+1}\right)}\left\|x_{n}\right\|+\frac{\left|\beta_{n+1}-\beta_{n}\right|}{1-\alpha_{n} \beta_{n+1} k_{n}\left(1-\xi_{n+1}\right)}\left\|y_{n}\right\| \\
& +\frac{\left|\beta_{n+1} \lambda_{n+1}-\beta_{n} \lambda_{n}\right|+\beta_{n+1}\left|\alpha_{n+1}-\alpha_{n}\right|}{1-\alpha_{n} \beta_{n+1} k_{n}\left(1-\xi_{n+1}\right)}\left\|x_{n+1}\right\| \\
& +\frac{\beta_{n+1}\left|\alpha_{n+1}-\alpha_{n}\right|}{1-\alpha_{n} \beta_{n+1} k_{n}\left(1-\xi_{n+1}\right)}\left\|T^{n+1}\left(\xi_{n+1} x_{n+1}+\left(1-\xi_{n+1}\right) x_{n+2}\right)\right\| \\
& +\frac{\alpha_{n} \beta_{n+1}}{1-\alpha_{n} \beta_{n+1} k_{n}\left(1-\xi_{n+1}\right)} \| T^{n+1}\left(\xi_{n+1} x_{n+1}+\left(1-\xi_{n+1}\right) x_{n+2}\right) \\
& -T^{n}\left(\xi_{n+1} x_{n+1}+\left(1-\xi_{n+1}\right) x_{n+2}\right) \| \\
\leqslant & \left(1-\sigma_{n}\right)\left\|x_{n+1}-x_{n}\right\|+M_{2}\left(\left|\lambda_{n+1}-\lambda_{n}\right|+\left|\beta_{n+1} \lambda_{n+1}-\beta_{n} \lambda_{n}\right|\right. \\
& \left.+2 \beta_{n+1}\left|\alpha_{n+1}-\alpha_{n}\right|+\left|\beta_{n+1}-\beta_{n}\right|+\alpha_{n} \beta_{n+1}\right) \\
\leqslant & \left(1-\sigma_{n}\right)\left\|x_{n+1}-x_{n}\right\|+M_{2}\left(2\left|\lambda_{n+1}-\lambda_{n}\right|+2\left|\alpha_{n+1}-\alpha_{n}\right|\right. \\
& \left.+2\left|\beta_{n+1}-\beta_{n}\right|+\alpha_{n} \beta_{n+1}\right)
\end{aligned}
$$

where

$$
\sigma_{n}=\frac{1-\left|\lambda_{n+1}-\beta_{n} \lambda_{n}\right|-\beta_{n+1}+\alpha_{n} \beta_{n+1}-\alpha_{n} \beta_{n+1} k_{n}\left(1-\xi_{n+1}+\xi_{n}\right)}{1-\alpha_{n} \beta_{n+1} k_{n}\left(1-\xi_{n+1}\right)}
$$

and $M_{2}>0$ is a constant such that for all $n \geqslant 1$,

$$
\begin{aligned}
M_{2} \geqslant\{ & \left\|x_{n}\right\|,\left\|y_{n}\right\|,\left\|T^{n+1}\left(\xi_{n+1} x_{n+1}+\left(1-\xi_{n+1}\right) x_{n+2}\right)\right\|, \\
& \left.\left\|T^{n+1}\left(\xi_{n+1} x_{n+1}+\left(1-\xi_{n+1}\right) x_{n+2}\right)-T^{n}\left(\xi_{n+1} x_{n+1}+\left(1-\xi_{n+1}\right) x_{n+2}\right)\right\|\right\} .
\end{aligned}
$$

By the assumptions (i)-(iv), we have

$$
\lim _{n \rightarrow \infty} \sigma_{n}=0, \quad \sum_{n=1}^{\infty} \sigma_{n}=\infty,
$$


and

$$
\begin{aligned}
& \sum_{n=1}^{\infty}\left(2\left|\lambda_{n+1}-\lambda_{n}\right|+2\left|\alpha_{n+1}-\alpha_{n}\right|+2\left|\beta_{n+1}-\beta_{n}\right|+\alpha_{n} \beta_{n+1}\right) \\
& \quad \leqslant 2 \sum_{n=1}^{\infty}\left|\lambda_{n+1}-\lambda_{n}\right|+4 \sum_{n=1}^{\infty} \alpha_{n}+2 \sum_{n=1}^{\infty}\left|\beta_{n+1}-\beta_{n}\right|+\sum_{n=1}^{\infty} \alpha_{n}<+\infty .
\end{aligned}
$$

Therefore, using Lemma 2.4 and (3.6), we obtain

$$
\left\|x_{n+1}-x_{n}\right\| \rightarrow 0, n \rightarrow \infty .
$$

Step 3. We prove that $\left\|x_{n}-T x_{n}\right\| \rightarrow 0, n \rightarrow \infty$.

From (3.1), we can easily have

$$
\left\|x_{n+1}-y_{n}\right\|=\left\|\left(1-\beta_{n}\right)\left(\lambda_{n} x_{n}\right)+\beta_{n} y_{n}-y_{n}\right\|=\left(1-\beta_{n}\right)\left\|\lambda_{n} x_{n}-y_{n}\right\|,
$$

then by the condition (i),

$$
\left\|x_{n+1}-y_{n}\right\| \rightarrow 0, \text { as } n \rightarrow \infty
$$

And

$$
\begin{aligned}
& \left\|y_{n}-T^{n}\left(\xi_{n} x_{n}+\left(1-\xi_{n}\right) x_{n+1}\right)\right\| \\
& =\left\|\left(1-\alpha_{n}\right) x_{n}+\alpha_{n} T^{n}\left(\xi_{n} x_{n}+\left(1-\xi_{n}\right) x_{n+1}\right)-T^{n}\left(\xi_{n} x_{n}+\left(1-\xi_{n}\right) x_{n+1}\right)\right\| \\
& =\left(1-\alpha_{n}\right)\left\|x_{n}-T^{n}\left(\xi_{n} x_{n}+\left(1-\xi_{n}\right) x_{n+1}\right)\right\| \\
& \leqslant\left(1-\alpha_{n}\right)\left\|x_{n}-x_{n+1}\right\|+\left(1-\alpha_{n}\right)\left\|x_{n+1}-T^{n}\left(\xi_{n} x_{n}+\left(1-\xi_{n}\right) x_{n+1}\right)\right\| .
\end{aligned}
$$

So from (3.9),

$$
\begin{aligned}
\left\|x_{n+1}-T^{n}\left(\xi_{n} x_{n}+\left(1-\xi_{n}\right) x_{n+1}\right)\right\|= & \left\|x_{n+1}-y_{n}+y_{n}-T^{n}\left(\xi_{n} x_{n}+\left(1-\xi_{n}\right) x_{n+1}\right)\right\| \\
\leqslant & \left\|x_{n+1}-y_{n}\right\|+\left\|y_{n}-T^{n}\left(\xi_{n} x_{n}+\left(1-\xi_{n}\right) x_{n+1}\right)\right\| \\
\leqslant & \left\|x_{n+1}-y_{n}\right\|+\left(1-\alpha_{n}\right)\left\|x_{n}-x_{n+1}\right\| \\
& +\left(1-\alpha_{n}\right)\left\|x_{n+1}-T^{n}\left(\xi_{n} x_{n}+\left(1-\xi_{n}\right) x_{n+1}\right)\right\|,
\end{aligned}
$$

which implies

$$
\left\|x_{n+1}-T^{n}\left(\xi_{n} x_{n}+\left(1-\xi_{n}\right) x_{n+1}\right)\right\| \leqslant \frac{1}{\alpha_{n}}\left\|x_{n+1}-y_{n}\right\|+\frac{1-\alpha_{n}}{\alpha_{n}}\left\|x_{n}-x_{n+1}\right\| .
$$

Then from (3.7) and (3.8),

$$
\left\|x_{n+1}-T^{n}\left(\xi_{n} x_{n}+\left(1-\xi_{n}\right) x_{n+1}\right)\right\| \rightarrow 0, \text { as } n \rightarrow \infty .
$$

Moreover, we have

$$
\begin{aligned}
\left\|x_{n}-T^{n} x_{n}\right\|= & \left\|x_{n}-x_{n+1}+x_{n+1}-T^{n}\left(\xi_{n} x_{n}+\left(1-\xi_{n}\right) x_{n+1}\right)+T^{n}\left(\xi_{n} x_{n}+\left(1-\xi_{n}\right) x_{n+1}\right)-T^{n} x_{n}\right\| \\
\leqslant & \left\|x_{n}-x_{n+1}\right\|+\left\|x_{n+1}-T^{n}\left(\xi_{n} x_{n}+\left(1-\xi_{n}\right) x_{n+1}\right)\right\| \\
& +\left\|T^{n}\left(\xi_{n} x_{n}+\left(1-\xi_{n}\right) x_{n+1}\right)-T^{n} x_{n}\right\| \\
\leqslant & \left\|x_{n}-x_{n+1}\right\|+\left\|x_{n+1}-T^{n}\left(\xi_{n} x_{n}+\left(1-\xi_{n}\right) x_{n+1}\right)\right\|+k_{n}\left(1-\xi_{n}\right)\left\|x_{n+1}-x_{n}\right\| .
\end{aligned}
$$

Then from (3.7) and (3.10), we have

$$
\left\|x_{n}-T^{n} x_{n}\right\| \rightarrow 0, \text { as } n \rightarrow \infty .
$$


Since $T$ is asymptotically nonexpansive, we derive that

$$
\begin{aligned}
\mid x_{n+1}-T x_{n+1} \| & =\left\|x_{n+1}-T^{n+1} x_{n+1}+T^{n+1} x_{n+1}-T x_{n+1}\right\| \\
& \leqslant\left\|x_{n+1}-T^{n+1} x_{n+1}\right\|+\left\|T^{n+1} x_{n+1}-T x_{n+1}\right\| \\
& \leqslant\left\|x_{n+1}-T^{n+1} x_{n+1}\right\|+k_{1}\left\|T^{n} x_{n+1}-x_{n+1}\right\| \\
& =\left\|x_{n+1}-T^{n+1} x_{n+1}\right\|+k_{1}\left\|T^{n} x_{n+1}-T^{n} x_{n}+T^{n} x_{n}-x_{n}+x_{n}-x_{n+1}\right\| \\
& =\left\|x_{n+1}-T^{n+1} x_{n+1}\right\|+k_{1}\left\|T^{n} x_{n+1}-T^{n} x_{n}\right\|+k_{1}\left\|T^{n} x_{n}-x_{n}\right\|+k_{1}\left\|x_{n}-x_{n+1}\right\| \\
& =\left\|x_{n+1}-T^{n+1} x_{n+1}\right\|+k_{1} k_{n}\left\|x_{n+1}-x_{n}\right\|+k_{1}\left\|T^{n} x_{n}-x_{n}\right\|+k_{1}\left\|x_{n}-x_{n+1}\right\| .
\end{aligned}
$$

So from (3.7) and (3.11), we obtain that

$$
\left\|x_{n}-T x_{n}\right\| \rightarrow 0, \text { as } n \rightarrow \infty .
$$

Step 4. We show that $x_{n} \rightarrow x^{*} \in F(T)$. Since $\left\{x_{n}\right\}$ is bounded, there exists a subsequence of $\left\{x_{n}\right\}$ which converges weakly to $x^{*}$, we assume that

$$
x_{n_{i}} \rightarrow x^{*} \in \mathrm{H},
$$

from (3.12) and the demiclosedness principle in Lemma 2.3, we have $q \in F(T)$.

For $n \geqslant 1$, let $z_{n}=\left(1-\beta_{n}\right) x_{n}+\beta_{n} y_{n}$, then from (3.1),

$$
x_{n+1}=z_{n}-\left(1-\beta_{n}\right)\left(1-\lambda_{n}\right) x_{n} .
$$

By the boundedness of $\left\{x_{n}\right\}$, and the condition (i), we have

$$
\left\|x_{n+1}-z_{n}\right\|=\left(1-\beta_{n}\right)\left(1-\lambda_{n}\right)\left\|x_{n}\right\| .
$$

By the (3.13) and (3.14), we can conclude that $z_{n_{i}} \rightarrow$ q. It follows that

$$
\begin{aligned}
x_{n+1} & =z_{n}-\left(1-\beta_{n}\right)\left(1-\lambda_{n}\right) x_{n} \\
& =\left(1-\left(1-\beta_{n}\right)\left(1-\lambda_{n}\right)\right) z_{n}-\left(1-\beta_{n}\right)\left(1-\lambda_{n}\right)\left(x_{n}-z_{n}\right) \\
& =\left(1-\left(1-\beta_{n}\right)\left(1-\lambda_{n}\right)\right) z_{n}-\left(1-\beta_{n}\right)\left(1-\lambda_{n}\right) \beta_{n}\left(x_{n}-y_{n}\right) .
\end{aligned}
$$

Also from Lemma 2.2, we have

$$
\begin{aligned}
\left\|z_{n}-x^{*}\right\|^{2} & =\left\|\left(1-\beta_{n}\right) x_{n}+\beta_{n} y_{n}-x^{*}\right\|^{2} \\
& =\left\|\left(x_{n}-x^{*}\right)-\beta_{n}\left(x_{n}-y_{n}\right)\right\|^{2} \leqslant\left\|x_{n}-x^{*}\right\|^{2}-2 \beta_{n}\left\langle x_{n}-y_{n}, z_{n}-x^{*}\right\rangle .
\end{aligned}
$$

By Lemma 2.2, (3.15), and (3.16), we have

$$
\begin{aligned}
\left\|x_{n+1}-x^{*}\right\|^{2}= & \|\left(1-\left(1-\beta_{n}\right)\left(1-\lambda_{n}\right)\right)\left(z_{n}-x^{*}\right) \\
& -\left(1-\beta_{n}\right)\left(1-\lambda_{n}\right) \beta_{n}\left(x_{n}-y_{n}\right)-\left(1-\beta_{n}\right)\left(1-\lambda_{n}\right) x^{*} \|^{2} \\
\leqslant & \left(1-\left(1-\beta_{n}\right)\left(1-\lambda_{n}\right)\right)^{2}\left\|z_{n}-x^{*}\right\| \\
& -2\left(1-\beta_{n}\right)\left(1-\lambda_{n}\right)\left\langle\beta_{n}\left(x_{n}-y_{n}\right)+x^{*}, x_{n+1}-x^{*}\right\rangle \\
\leqslant & \left(1-\left(1-\beta_{n}\right)\left(1-\lambda_{n}\right)\right)^{2}\left\|z_{n}-x^{*}\right\| \\
& -2\left(1-\beta_{n}\right)\left(1-\lambda_{n}\right) \beta_{n}\left\langle x_{n}-y_{n}, x_{n+1}-x^{*}\right\rangle-2\left(1-\beta_{n}\right)\left(1-\lambda_{n}\right)\left\langle x^{*}, x_{n+1}-x^{*}\right\rangle \\
\leqslant & \left(1-\left(1-\beta_{n}\right)\left(1-\lambda_{n}\right)\right)\left(\left\|x_{n}-x^{*}\right\|^{2}-2 \beta_{n}\left\langle x_{n}-y_{n}, z_{n}-x^{*}\right\rangle\right) \\
& -2\left(1-\beta_{n}\right)\left(1-\lambda_{n}\right) \beta_{n}\left\langle x_{n}-y_{n}, x_{n+1}-x^{*}\right\rangle-2\left(1-\beta_{n}\right)\left(1-\lambda_{n}\right)\left\langle x^{*}, x_{n+1}-x^{*}\right\rangle \\
\leqslant & \left(1-\delta_{n}\right)\left\|x_{n}-x^{*}\right\|^{2}+\delta_{n}\left(-2 \beta_{n}\left\langle x_{n}-y_{n}, z_{n}-x^{*}\right\rangle\right. \\
& \left.-2 \beta_{n}\left\langle x_{n}-y_{n}, x_{n+1}-x^{*}\right\rangle-2\left\langle x^{*}, x_{n+1}-x^{*}\right\rangle\right) \\
= & \left(1-\delta_{n}\right)\left\|x_{n}-x^{*}\right\|^{2}+\delta_{n} \gamma_{n},
\end{aligned}
$$


where

$$
\delta_{n}=\left(1-\beta_{n}\right)\left(1-\lambda_{n}\right), \quad \gamma_{n}=-2 \beta_{n}\left\langle x_{n}-y_{n}, z_{n}-x^{*}\right\rangle-2 \beta_{n}\left\langle x_{n}-y_{n}, x_{n+1}-x^{*}\right\rangle-2\left\langle x^{*}, x_{n+1}-x^{*}\right\rangle .
$$

By the conditions (i) and (iii), we can easily have that

$$
\lim _{n \rightarrow \infty} \delta_{n}=0, \quad \sum_{n=1}^{\infty} \delta_{n}=+\infty
$$

Because

$$
\limsup _{n \rightarrow \infty}-2 \beta_{n}\left\langle x_{n}-y_{n}, z_{n}-x^{*}\right\rangle=0, \quad \limsup _{n \rightarrow \infty}-2 \beta_{n}\left\langle x_{n}-y_{n}, x_{n+1}-x^{*}\right\rangle=0,
$$

and also

$$
\limsup _{n \rightarrow \infty}-2\left\langle x^{*}, x_{n+1}-x^{*}\right\rangle=\lim _{i \rightarrow \infty}-2\left\langle x^{*}, x_{n_{i}}-x^{*}\right\rangle=-2\left\langle x^{*}, q-x^{*}\right\rangle \leqslant 0 .
$$

So

$$
\limsup _{n \rightarrow \infty} \gamma_{n} \leqslant 0
$$

Therefore, applying Lemma 2.4 to (3.17), we conclude that

$$
\lim _{n \rightarrow \infty}\left\|x_{n}-x^{*}\right\|=0
$$

which completes this proof.

Remark 3.2. In algorithm (3.1), the iterative coefficients $\alpha_{n}, \beta_{n}, \lambda_{n}$ are available.

For example, take $\alpha_{n}=\frac{1}{n^{2}}, \beta_{n}=\lambda_{n}=1-\frac{1}{\sqrt{n}}$, then

$$
\sum_{n=1}^{\infty} \alpha_{n}=\sum_{n=1}^{\infty} \frac{1}{n^{2}}<\infty, \quad \sum_{n=1}^{\infty}\left(1-\lambda_{n}\right)\left(1-\beta_{n}\right)=\sum_{n=1}^{\infty} \frac{1}{n}=\infty .
$$

From

$$
\left|\lambda_{n+1}-\lambda_{n}\right|=\left|\left(1-\frac{1}{\sqrt{n+1}}\right)-\left(1-\frac{1}{\sqrt{n}}\right)\right|=\frac{1}{\sqrt{n}}-\frac{1}{\sqrt{n+1}}=\frac{1}{\sqrt{n(n+1)}(\sqrt{n}+\sqrt{n+1})} \leqslant \frac{1}{n^{\frac{3}{2}}},
$$

we have

$$
\sum_{n=1}^{\infty}\left|\lambda_{n+1}-\lambda_{n}\right| \leqslant \sum_{n=1}^{\infty} \frac{1}{n^{\frac{3}{2}}}<\infty
$$

and also

$$
\sum_{n=1}^{\infty}\left|\beta_{n+1}-\beta_{n}\right|<\infty
$$

Because

$$
1-\frac{1}{\sqrt{n+1}} \geqslant\left(1-\frac{1}{\sqrt{n}}\right)^{2}
$$

then

$$
\left|\left(1-\frac{1}{\sqrt{n+1}}\right)-\left(1-\frac{1}{\sqrt{n}}\right)^{2}\right|=\left(1-\frac{1}{\sqrt{n+1}}\right)-\left(1-\frac{1}{\sqrt{n}}\right)^{2} .
$$

We want to know that

$$
\left|\lambda_{n+1}-\beta_{n} \lambda_{n}\right|+\beta_{n+1}=\left|\left(1-\frac{1}{\sqrt{n+1}}\right)-\left(1-\frac{1}{\sqrt{n}}\right)^{2}\right|+1-\frac{1}{\sqrt{n+1}} \leqslant 1,
$$


which is equivalent to

$$
\begin{aligned}
& 1-\frac{1}{\sqrt{n+1}}-1+\frac{2}{\sqrt{n}}-\frac{1}{n}+1-\frac{1}{\sqrt{n+1}}-1 \leqslant 0, \\
& \frac{2}{\sqrt{n}}-\frac{1}{n}-\frac{2}{\sqrt{n+1}} \leqslant 0, \\
& \frac{2}{\sqrt{n}}-\frac{2}{\sqrt{n+1}} \leqslant \frac{1}{n} \\
& \frac{2 n}{\sqrt{n(n+1)}(\sqrt{n+1}+\sqrt{n})} \leqslant 1 \text {. }
\end{aligned}
$$

Therefore

$$
\frac{2 n}{\sqrt{n(n+1)}(\sqrt{n+1}+\sqrt{n})} \leqslant \frac{2 n}{2 n \sqrt{n}}=\frac{1}{\sqrt{n}} \leqslant 1 .
$$

Corollary 3.3. Let $\mathrm{C} \subset \mathrm{H}$ be a nonempty bounded convex closed set of a Hilbert space $\mathrm{H}$ and $\theta \in \mathrm{C}$. Let $\mathrm{T}: \mathrm{C} \rightarrow \mathrm{C}$ be a asymptotically nonexpansive mapping with a sequence $\left\{k_{n}\right\}$ such that $\mathrm{F}(\mathrm{T}) \neq \emptyset$. Suppose $\left\{\alpha_{\mathrm{n}}\right\},\left\{\beta_{\mathrm{n}}\right\},\left\{\lambda_{\mathrm{n}}\right\},\left\{\xi_{\mathrm{n}}\right\}$ are real number sequences in $(0,1)$. Let $\left\{x_{n}\right\}$ be generated by

$$
\left\{\begin{array}{l}
x_{1} \in C \\
y_{n}=\left(1-\alpha_{n}\right) x_{n}+\alpha_{n} T^{n}\left(\frac{x_{n}+x_{n+1}}{2}\right) \\
x_{n+1}=\left(1-\beta_{n}\right)\left(\lambda_{n} x_{n}\right)+\beta_{n} y_{n}
\end{array}\right.
$$

Suppose the following conditions are satisfied:
(i) $\lim _{n \rightarrow \infty} \beta_{n}=1, \lim _{n \rightarrow \infty} \lambda_{n}=1$;
(ii) $\sum_{n=1}^{\infty} \alpha_{n}<+\infty, \sum_{n=1}^{\infty}\left|\lambda_{n+1}-\lambda_{n}\right|<+\infty, \sum_{n=1}^{\infty}\left|\beta_{n+1}-\beta_{n}\right|<+\infty$;
(iii) $\sum_{n=1}^{\infty}\left(1-\lambda_{n}\right)\left(1-\beta_{n}\right)=+\infty,\left|\lambda_{n+1}-\beta_{n} \lambda_{n}\right|+\beta_{n+1} \leqslant 1$;
(iv) $\sum_{n=1}^{\infty} \sup _{x \in C}\left\|T^{n+1} x-T^{n} x\right\|<+\infty$.

Then the sequence $\left\{x_{n}\right\}$ converges strongly to a fixed point $x^{*} \in F(T)$.

Proof. Let $\xi_{n}=\frac{1}{2}$, then by the proof of Theorem 3.1, we can easily prove the sequence $\left\{x_{n}\right\}$ generated by (3.18) converges strongly to a point $x^{*} \in F(T)$.

Corollary 3.4. Let $\mathrm{C} \subset \mathrm{H}$ be a nonempty bounded convex closed set of a Hilbert space $\mathrm{H}$ and $\theta \in \mathrm{C}$. Let $\mathrm{T}: \mathrm{C} \rightarrow \mathrm{C}$ be a asymptotically nonexpansive mapping with a sequence $\left\{k_{n}\right\}$ such that $\mathrm{F}(\mathrm{T}) \neq \emptyset$. Suppose $\left\{\alpha_{n}\right\},\left\{\beta_{n}\right\},\left\{\xi_{n}\right\}$ are real number sequences in $(0,1)$. Let $\left\{x_{n}\right\}$ be generated by

$$
\left\{\begin{array}{l}
x_{1} \in C \\
y_{n}=\left(1-\alpha_{n}\right) x_{n}+\alpha_{n} T^{n}\left(\xi_{n} x_{n}+\left(1-\xi_{n}\right) x_{n+1}\right) \\
x_{n+1}=\left(1-\beta_{n}\right) u+\beta_{n} y_{n} .
\end{array}\right.
$$

Suppose the following conditions are satisfied:

(i) $\lim _{n \rightarrow \infty} \beta_{n}=1, \lim _{n \rightarrow \infty} \xi_{n}=1$;

(ii) $\sum_{n=1}^{\infty} \alpha_{n}<+\infty, \sum_{n=1}^{\infty}\left|\beta_{n+1}-\beta_{n}\right|<+\infty$;

(iii) $\sum_{n=1}^{\infty} \sup _{x \in C}\left\|T^{n+1} x-T^{n} x\right\|<+\infty$.

Then the sequence $\left\{x_{n}\right\}$ converges strongly to a fixed point $x^{*} \in F(T)$.

Proof. By the proof of Theorem 3.1, we can easily prove the sequence $\left\{x_{n}\right\}$ generated by the (3.19) converges strongly to a point $x^{*} \in F(T)$. 
Remark 3.5. From our theorem and corollaries we know the algorithm (3.1) is implicit iterative algorithm. When $\xi_{n}=\frac{1}{2}$, and $\mathrm{T}$ is a nonexpansive mapping, Theorem 3.1 can be reduced to the main result of $\mathrm{Xu}$ (see [19]). When $\xi_{n}=1$, and $T$ is a nonexpansive mapping, Theorem 3.1 can be reduced to the main result of Fan et al. (see [3]).

\section{Applications}

Lemma 4.1 ([20]). Let $\mathrm{C} \subset \mathrm{H}$ be a nonempty convex closed set of a Hilbert space $\mathrm{H}$ and $\theta \in \mathrm{C}$. Let $\mathrm{U}_{i}: \mathrm{C} \rightarrow \mathrm{H}$ be $\mu_{i}$-inverse strongly monotone mapping. Let $\mathrm{P}_{\mathrm{C}}$ denote the orthogonal projection onto the set $\mathrm{C}$. Let $0<\gamma_{i}<$ $\mu_{i}, i=1,2, \ldots, t$, where $t$ is a positive integer. Let

$$
S \triangleq P_{C}\left(I-\gamma_{t} U_{t}\right) P_{C}\left(I-\gamma_{t-1} U_{t-1}\right) \cdots P_{C}\left(I-\gamma_{1} U_{1}\right)
$$

Then $\mathrm{S}$ is a attracting mapping and $\mathrm{F}(\mathrm{S})=\bigcap_{i=1}^{\mathrm{t}} \mathrm{N}\left(\mathrm{U}_{i}\right)$.

Theorem 4.2. Let $\mathrm{C} \subset \mathrm{H}$ be a nonempty bounded convex closed set of a Hilbert space $\mathrm{H}$ and $\theta \in \mathrm{C}$. Let $\mathrm{U}_{i}: \mathrm{C} \rightarrow \mathrm{H}$ be $\mu_{i}$-inverse strongly monotone. Suppose $\left\{\alpha_{n}\right\},\left\{\beta_{n}\right\},\left\{\lambda_{n}\right\},\left\{\xi_{n}\right\}$ are real number sequences in $(0,1)$. Let $\left\{x_{n}\right\}$ be generated by

$$
\left\{\begin{array}{l}
x_{1} \in C \\
y_{n}=\left(1-\alpha_{n}\right) x_{n}+\alpha_{n} S\left(\xi_{n} x_{n}+\left(1-\xi_{n}\right) x_{n+1}\right) \\
x_{n+1}=\left(1-\beta_{n}\right)\left(\lambda_{n} x_{n}\right)+\beta_{n} y_{n} .
\end{array}\right.
$$

Suppose the following conditions are satisfied:

(i) $\lim _{n \rightarrow \infty} \beta_{n}=1, \lim _{n \rightarrow \infty} \lambda_{n}=1, \lim _{n \rightarrow \infty} \xi_{n}=1$;

(ii) $\sum_{n=1}^{\infty} \alpha_{n}<+\infty, \sum_{n=1}^{\infty}\left|\lambda_{n+1}-\lambda_{n}\right|<+\infty, \sum_{n=1}^{\infty}\left|\beta_{n+1}-\beta_{n}\right|<+\infty$;

(iii) $\sum_{n=1}^{\infty}\left(1-\lambda_{n}\right)\left(1-\beta_{n}\right)=+\infty,\left|\lambda_{n+1}-\beta_{n} \lambda_{n}\right|+\beta_{n+1} \leqslant 1$

Then the sequence $\left\{x_{n}\right\}$ converges strongly to a common element $\hat{x} \in \bigcap_{i=1}^{t} N\left(U_{i}\right)$.

Proof. From Lemma 4.1 and Theorem 3.1, we can easily prove the sequence $\left\{x_{n}\right\}$ converges strongly to a point $\hat{x} \in \bigcap_{i=1}^{t} N\left(u_{i}\right)$.

Theorem 4.3. Let $\mathrm{C} \subset \mathrm{H}$ be a nonempty bounded convex closed set of a Hilbert space $\mathrm{H}$ and $\theta \in \mathrm{C}$. Let $\mathrm{U}_{\mathrm{i}}: \mathrm{C} \rightarrow \mathrm{H}$ be $\mu_{i}$-inverse strongly monotone. Suppose $\left\{\alpha_{n}\right\},\left\{\beta_{n}\right\},\left\{\lambda_{n}\right\},\left\{\xi_{n}\right\}$ are real number sequences in $(0,1)$. Let $\left\{x_{n}\right\}$ be generated by

$$
\left\{\begin{array}{l}
x_{1} \in C \\
y_{n}=\left(1-\alpha_{n}\right) x_{n}+\alpha_{n} S\left(\xi_{n} x_{n}+\left(1-\xi_{n}\right) x_{n+1}\right) \\
x_{n+1}=\left(1-\beta_{n}\right) u+\beta_{n} y_{n} .
\end{array}\right.
$$

Suppose the following conditions are satisfied:

(i) $\lim _{n \rightarrow \infty} \beta_{n}=1, \lim _{n \rightarrow \infty} \lambda_{n}=1, \lim _{n \rightarrow \infty} \xi_{n}=1$;

(ii) $\sum_{n=1}^{\infty} \alpha_{n}<+\infty, \sum_{n=1}^{\infty}\left|\lambda_{n+1}-\lambda_{n}\right|<+\infty, \sum_{n=1}^{\infty}\left|\beta_{n+1}-\beta_{n}\right|<+\infty$;

(iii) $\sum_{n=1}^{\infty}\left(1-\lambda_{n}\right)\left(1-\beta_{n}\right)=+\infty,\left|\lambda_{n+1}-\beta_{n} \lambda_{n}\right|+\beta_{n+1} \leqslant 1$ 
Then the sequence $\left\{x_{n}\right\}$ converges strongly to a common element $\hat{x} \in \bigcap_{i=1}^{t} N\left(U_{i}\right)$.

Proof. From Theorem 3.1, Lemma 4.1, and the Theorem 1 of [5], we can easily prove the sequence $\left\{x_{n}\right\}$ converges strongly to a point $\hat{x} \in \bigcap_{i=1}^{t} N\left(U_{i}\right)$.

\section{Acknowledgment}

This work was supported by the National Natural Science Foundation of China (Grant no. 11671365) and the Natural Science Foundation of Zhejiang Province (Grant no. LY14A010011).

\section{References}

[1] E. Blum, W. Oettli, From optimization and variational inequalities to equilibrium problems, Math. Student, 63 (1994), 123-145. 1

[2] L.-C. Ceng, C.-Y. Wang, J.-C. Yao, Strong convergence theorems by a relaxed extragradient method for a general system of variational inequalities, Math. Methods Oper. Res., 67 (2008), 375-390. 1

[3] Q. Fan, Z. Yao, Strong convergence theorems for a nonexpansive mapping and its applications for solving the split feasibility problem, J. Nonlinear Sci. Appl., 10 (2017), 1470-1477. 1, 3.5

[4] S. D. Flåm, A. S. Antipin, Equilibrium programming using proximal-like algorithms, Math. Programming, 78 (1996), 29-41. 1

[5] T.-H. Kim, H.-K. Xu, Strong convergence of modified Mann iterations, Nonlinear Anal., 61 (2005), 51-60. 4

[6] G. López, V. Martín-Márquez, F. Wang, H.-K. Xu, Forward-backword splitting methods for accretive operators in Banach spaces, Abstr. Appl. Anal., 2012 (2012), 25 pages. 2.2

[7] J. Lou, L.-J. Zhang, Z. He, Viscosity approximation methods for asymptotically nonexpansive mappings, Appl. Math. Comput., 203 (2008), 171-177. 1

[8] A. Moudafi, Viscosity approximation methods for fixed points problems, J. Math. Anal. Appl., 241 (2000), 46-55. 1

[9] K. Nakajo, W. Takahashi, Strong convergence theorems for nonexpansive mappings and nonexpansive semigroups, J. Math. Anal. Appl., 279 (2003), 372-379. 1

[10] J.-W. Peng, J.-C. Yao, A viscosity approximation scheme for system of equilibrium problems, nonexpansive mappings and monotone mappings, Nonlinear Anal., 71 (2009), 6001-6010.

[11] J.-W. Peng, J.-C. Yao, Strong convergence theorems of iterative scheme based on the extra gradient method for mixed equilibrium problems and fixed point problems, Math. Comput. Modelling, 49 (2009), 1816-1828.

[12] X. Qin, Y. J. Cho, S. M. Kang, Viscosity approximation methods for generalized equlibrium problems and fixed point problems with applications, Nonlinear Anal., 72 (2010), 99-112.

[13] Y. Song, R. Chen, H. Zhou, Viscosity approximation methods for nonexpansive mapping sequences in Banach spaces, Nonlinear Anal., 66 (2007), 1016-1024.

[14] P. Sunthrayuth, P. Kumam, Viscosity approximation methods base on generalized contraction mappings for a countable family of strict pseudo-contractions, a general system of variational inequalities and a generalized mixed equilibrium problem in Banach spaces, Math. Comput. Modelling, 58 (2013), 1814-1828. 1

[15] Y.-H. Wang, Y.-H. Xia, Strong convergence for asymptotical pseudocontractions with the demiclosedness principle in banach spaces, Fixed Point Theory Appl., 2012 (2012), 8 pages. 2.3

[16] H.-K. Xu, Iterative algorithms for nonlinear operators, J. London Math. Soc., 66 (2002), 240-256. 2.4

[17] H.-K. Xu, An iterative approach to quadratic optimization, J. Optim. Theory Appl., 116 (2003), 659-678. 2.4

[18] H.-K. Xu, Viscosity approximation methods for nonexpansive mappings, J. Math. Anal. Appl., 298 (2004), 279-291. 1

[19] H.-K. Xu, M. A. Aoghamdi, N. Shahzad, The viscosity technique for the implicit midpoint rule of nonexpansive mappings in Hilbert spaces, Fixed Point Theory Appl., 2015 (2015), 12 pages. 1, 3.5

[20] Q. Yan, G. Cai, Convergence analysis of modified viscosity implicit rules of asymptotically nonexpansive mappings in Hilbert spaces, Revista de la Real Academia de Ciencias Exactas, Fasicas y Naturales. Serie A. Matematicas (RACSAM), 2017 (2017), 16 pages. 4.1

[21] Y. Yao, Y.-C. Liou, R. Chen, Strong convergence of an iterative algorithm for pseudocontractive mapping in Banach spaces, Nonlinear Anal., 67 (2007), 3311-3317. 1

[22] Y. Yao, Y.-C. Liou, S. M. Kang, Approach to common elements of variational inequality problems and fixed point problems via a relaxed extragradient method, Comput. Math. Appl., 59 (2010), 3472-3480.

[23] Y. Yao, M. A. Noor, Y.-C. Liou, S. M. Kang, Some new algorithms for solving mixed equilibrium problems, Comput. Math. Appl., 60 (2010), 1351-1359.

[24] Y. Yao, N. Shahzad, Y.-C. Liou, Modified semi-implicit midpoint rule for nonexpansive mappings, Fixed Point Theory Appl., 2015 (2015), 15 pages. 1 